Research Article

\title{
Re-evaluating the efficacy and toxicity of procarbazine, limestone and vincristine (PCV) in relapsed glioblastoma in the concurrent/adjuvant temozolamide era
}

\begin{abstract}
Background: Publication of the Stupp Trial in 2005 led to adoption of concurrent/adjuvant Temozolomide(TMZ) with radiotherapy as the standard of care for fit patients with newly diagnosed Glioblastoma. No accepted standard treatment exists for relapsed disease. However, nitrosurea based regimens are commonly used. Little published evidence exists for the use of such nitrosurea based regimens in the post-Stupp era.

Patients and Method: We present a retrospective study of a cohort of patients between 2009 and 2014 treated with procarbazine, lomustine and vincristine (PCV) for disease progression after completing treatment with the Stupp regime.

Result: 33 patients received PCV chemotherapy having previously been treated with the Stupp regime. Median age was 47 (range 23 to 74). The median number of cycles received was 2 (range 1 to 6). Patients underwent magnetic resonance imaging (MRI) to assess response: $19 \%, 23 \%$ \& 58\% showed Partial Response, Stable, and Progressive Disease respectively. The Median Overall Survival (OS) was 26weeks. Median Progression Free Survival (PFS) was 17weeks with Progression Free Survival at 6 months (PFS 6) of $20 \%$. Grade $3 / 4$ haematological toxicity was observed in $31 \%$ of patients, of these, $18 \%$ developed grade $3 / 4$ thrombocytopenia. Non haematological toxicities included venous thromboembolism in $13 \%$ of cases. Treatment discontinuation due to toxicities occurred in $6 \%$ of patients.
\end{abstract}

Conclusion: Our experience in this cohort of patients suggests that PCV in relapsed GBM following the Stupp regimen provides modest survival benefit but is relatively well tolerated with a low risk of serious toxicity.

Keywords: glioblastoma, recurrent, pcv, temozolamide, chemotherapy
Volume 6 Issue 3 - 2016

Das P, McCaffrey S, Conkey D S

Northern Ireland Cancer Centre, Belfast City Hospital, UK

Correspondence: Prantik Das, Northern Ireland Cancer Centre, Belfast City Hospital, UK, Email drpran-tik@yahoo.co.uk

Received: October 31, 2016 | Published: December 09, 2016

\section{Introduction}

Glioblastoma Multiforme (GBM) is the most frequently occurring primary brain tumour, accounting for $51.2 \%$ of all newly diagnosed gliomas and $36 \%$ of all cerebral tumours. ${ }^{1}$ Despite current optimal treatment long-term prognosis remains poor. Median overall survival is 15 months, and in only a small number of long-term survivors (2$5 \%$ ) does survival time exceed 3years. ${ }^{2}$ Poor outcomes are in part attributable to the highly aggressive nature of GBM. ${ }^{3}$ These tumours are naturally infiltrative and residual disease is almost always present following surgery, ${ }^{1}$ as such, the majority of patients relapse following first line treatment for newly diagnosed disease. Tumour progression after first-line treatment typically occurs after 20-27weeks. ${ }^{4}$

Historically nitrosurea based chemotherapy regimens combined with radiotherapy made up the standard of care for newly diagnosed GBM. Nitrosureas were frequently used due to their liposolubility, enabling these compounds to cross the blood brain barrier. ${ }^{1,5}$ Although recognised as the most active chemotherapeutic agent in this setting, the use of nitrosurea based treatments remained controversial with trials failing to demonstrate superior activity of PCV when added to radiotherapy compared with radiotherapy alone. ${ }^{2}$ Publication of the landmark Stupp Trial ${ }^{3}$ in 2005 clearly demonstrated survival benefit with less toxicity using the alkylating agent Temozolomide (TMZ) in combination with radiotherapy. ${ }^{4}$ This led to concurrent/adjuvant TMZ with RT becoming the standard of care for fit patients with newly diagnosed GBM. Widespread use of TMZ in patients with newly diagnosed GBM led to re-evaluation of nitrosureas at progression or recurrence. ${ }^{6,7}$ Little published evidence exists for the use of such regimens in the post-Stupp era. As a result no accepted standardised treatment exists for recurrent GBM. Salvage chemotherapy remains the most commonly utilised treatment option, ${ }^{8}$ of which, nitrosurea based regimens predominate. Here we report the findings of a retrospective study of our regional centre's experience of PCV as salvage chemotherapy for relapsed/recurrent GBM.

\section{Patients and method}

Between April 2009 and March 2014, 44 patients with recurrent GBM received salvage PCV, and of these, 33 had received the Stupp regime as first line treatment. Treatment was administered at 6 weekly intervals (Procarbazine 100mg/m2 D1-D10, Lomustine 100mg/m2 D1 and Vincristine $1.5 \mathrm{mg} / \mathrm{m} 2$ with maximum dose of $2 \mathrm{mg}$ ). A maximum of 6 cycles were offered until patients had disease progression or experienced unacceptable toxicities. Patients underwent magnetic resonance imaging (MRI) to assess response after 2 to 3 cycles of chemotherapy. 
We identified all patients who received PCV chemotherapy from our electronic pharmacy data base. Subsequent systematic analysis of an in house Oncology data network, individual patients medical records and relevant imaging systems allowed collation of relevant data including patient demographics, previous treatment including those treated with the Stupp regime, performance status at time of treatment and any toxicities experienced. Diagnosis of recurrence was confirmed by means of neuroimaging or histological analysis in patients who underwent resection. Imaging response to treatment was assessed using McDonald's criteria. ${ }^{9}$ Toxicities were classified according to the Common Terminology Criteria for Adverse Event (CTCAE) version 3.0.

Progression free survival (PFS) was measured from initiation of systemic therapy until disease progression by clinical assessment or neuroimaging. The median Overall Survival (OS) was measured from the start of chemotherapy to death from any cause or to last documented follow up. SPSS software was used for statistical analysis.

\section{Results}

\section{Patient demographics}

Demographic analysis of the thirty-three patients who received treatment with the Stupp regime prior to recurrence of their disease confirmed 19 male and 14 female patients. All 33 patients completed concurrent chemoradiotherapy. Only 10 patients however completed a total of 6 cycles of adjuvant Temozolamide. The number of cycles received is shown in Table 1. Median age was 47years (range 23 to 74). Median number of cycles of PCV chemotherapy received was 2 (range 1 to 6 ). Four patients $(12 \%)$ received further palliative Temozolamide chemotherapy after disease progression on PCV. Five patients $(16 \%)$ had repeat resection at relapse prior to treatment with PCV.

Table I Demonstrates patient characteristics

\begin{tabular}{lll}
\hline & No of Patients & Percentage \\
\hline Median Age & 47 & \\
Age Range & $23-74$ & 58 \\
Male & 19 & 42 \\
Female & 14 & 100 \\
Previous chemoradiotherapy & 33 & \\
Adjuvant Temozolamide & & 28 \\
I cycles & 9 & 12 \\
2 cycles & 4 & 6 \\
3 cycles & 2 & 12 \\
4 cycles & 4 & 12 \\
5 cycles & 4 & 30 \\
6 cycles & 10 & \\
WHO Performance Status & & 60 \\
0-I & 20 & 30 \\
2 & 10 & 10 \\
4-Mar & 3 & 12 \\
2nd line temozolamide after relapse & 4 & 16 \\
Surgery at relapse prior to PCV & 5 & \\
\hline
\end{tabular}

\section{Toxicities}

Ten patients (31\%) developed CTCAE grade 3/4 haematological toxicity: 6 patients $(18 \%)$ had thrombocytopenia. No case of haemorrhage was identified. Transfusion of packed red cells was required in 2 patients and a further 2 patients required platelet transfusion. Only one patient developed neutropenic sepsis. No patients required cytokine support. Two patients $(6 \%)$ had nonneutropenic infection. Non-haematological toxicity comprised of 4 cases $(13 \%)$ of venous thromboembolism: one pulmonary embolism and 3 lower limb deep vein thromboses. One patient developed interstitial lung disease, probably secondary to Lomustine, which was demonstrated with high resolution computed tomography (CT) scan. An increase in liver enzymes was documented in 2 patients but no interruption in treatment was required. Chemotherapy was stopped in 2 patients because of serious toxicity: one had neutropenic infection and another developed interstitial lung disease. No chemotherapy related deaths were identified.

\section{Efficacy}

Forty four patients received PCV chemotherapy among them 33 had previously received Stupp regime. Eleven patients had not received the standard concurrent chemoradiotherapy as these patients were diagnosed at a time before the Stupp regime was accepted as the recognised standard of care for newly diagnosed GBM at our institution. These patients are not considered further. The median number of cycles received was 2 (range 1 to 6 ). Nine patients received one cycle, four patients 2 cycles, two patients 3 cycles, four patients 4 cycles, four patients 5 cycles and ten patients 6 cycles respectively. Patients underwent repeat MRI to assess response to treatment. MRI demonstrated a partial response in $19 \%$ patients, $23 \%$ had stable disease and $58 \%$ had progressive disease. No patients had a complete response. The median Overall Survival (OS) measured from start of chemotherapy was 26weeks. Median Progression Free Survival (PFS) was 17 weeks with a Progression Free Survival at 6months (PFS 6) of $20 \%$. Patients who underwent re-resection following relapse had significantly better survival outcomes (median Overall Survival 48weeks) (Figures $1 \& 2$ ).

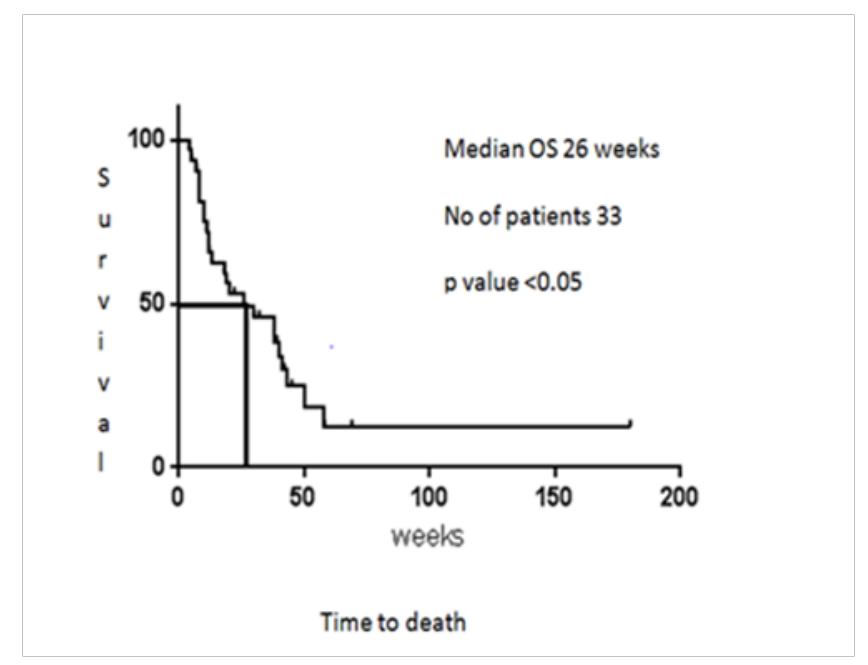

Figure I Kaplan-Meier Estimates of Overall survival.

\section{Discussion}

Following the land mark Stupp trial in $2005,{ }^{3} \mathrm{TMZ}$ with concurrent radiotherapy became widely accepted as the gold standard for first line therapy for newly diagnosed glioblastoma. There remains however no standard of care for recurrent or relapsed disease. Nitrosourea based chemotherapy regimens remain the most universally accepted salvage treatment option. 


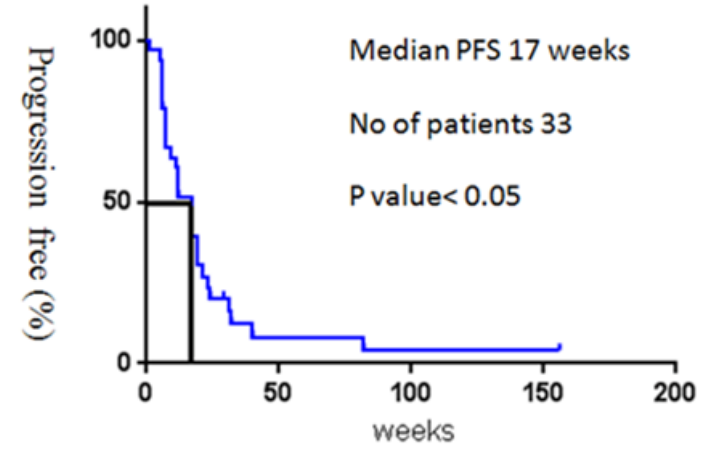

Time to progression

Figure 2 Kaplan-Meier Estimates of Progression free survival.

In our study, treatment of relapsed/recurrent GBM with salvage PCV chemotherapy was associated with a median progression free survival of 17 weeks and PFS $620 \%$. Median overall survival from the initiation of PCV chemotherapy was 26 weeks. All patients included in the analysis had received treatment with concurrent TMZ and RT at initial diagnosis. Similar results were reported in a recent publication by Kuhnhenn et al., ${ }^{10}$ of a single institution retrospective evaluation of combination PBV (Procarbazine, Carmustine and Vincristine) in the treatment of 57 patients with recurrent glioblastoma. Median progression free survival of 15 weeks and PFS 6 of $21 \%$ is documented and is in line with the findings of our study. Comparable with our study all patients had previously received treatment with TMZ chemotherapy but a proportion of patients received TMZ in combination with other chemotherapeutic agents. As a result PBV was used in some patients as third line salvage treatment. The lack of standardised inclusion criteria in this series complicates direct comparison with our study. Additionally, the authors of this study experienced difficulty in determining exact timing of progression in some cases due to difficulty with documented follow up given the retrospective nature of the analysis.

Another 2008 retrospective case note review of 32 recurrent/ progressive GBM patients by Happold et al., ${ }^{7}$ with ACNU alone or in combination with Teniposide or Cytarabine showed median progression free survival from start of ACNU of 2.7 months and a median OS of 6.7 months with PFS 6 of $20 \%$. Survival at 12 months was documented as $26 \%$ which again is comparable with our experience. No association was found between age, Karnofsky Performance Status (KPS), dose of steroids, re-resection and time to progression or survival. Several phase II studies have demonstrated equivocal efficacy of nitrosurea based regimens, noting similar PFS 6 and median OS rates. Indeed in 2008, a large Meta-analysis by Lamborn et al., ${ }^{11}$ collating data from several such studies reported a median survival of 30 weeks and a PFS 6 of around $16 \%$ with salvage chemotherapy in recurrent glioblastoma, with a 1-year survival rate of $25 \%$. This study suggested that progression status at 6 months was a strong predictor of survival and therefore a valid end point for trials of therapy for recurrent malignant glioma.

A phase II trial carried out in 2004 by Brandes et al. ${ }^{12}$ reported a PFS 6 of $42 \%$ in patients with recurrent glioblastoma treated with a BCNU regimen. In this study, however, it should be noted that patients were all chemotherapy naive which may account for the marked difference between the results seen here compared with the consensus of the studies noted above. In a similar vein several studies have suggested superior results with salvage chemotherapy. One such retrospective analysis published in 2006 by Shmidt et al., ${ }^{13}$ demonstrated a median time to progression of 17.1 weeks and a PFS 6 of $38.4 \%$ with PCV in recurrent glioblastoma (median survival from start of PCV of 34.3 weeks). $62 \%$ of patients in this study had previous chemotherapy but only $15 \%$ had prior TMZ. Also significantly prolonged survival was found in patients who underwent surgical re-resection at relapse which is similar to our findings. The authors admit that the lack of standard assessment may have resulted in an overestimation of the benefit from PCV. Additionally patients included in this study had favourable prognostic factors. Direct comparison between our study, which assesses PCV after initial treatment with TMZ/RT, and studies from the pre-Stupp era is not logical.

Recognised risk factors in recurrent GBM may play a role in the varied response seen in the literature to nitrosurea based chemotherapy regimens, with study populations where more favourable prognostic factors predominate tending to better outcomes specifically overall survival rate. A retrospective pooled analysis ${ }^{14}$ reviewed responses to nitrosurea based chemotherapy in patients pre-treated with TMZ in an attempt to explain the varied response seen in the literature and suggested that time from radiotherapy to initiation of treatment and re-resection at relapse were predictive of the clinical benefit. In our study $16 \%$ had re-resection. This group of patients had significantly better survival (Median OS 48weeks).

The EORTC brain cancer group retrospectively analysed data from 300 patients with recurrent GBM, from phase I or II trials, to determine prognostic factors and predictive tools for recurrence..$^{15}$ This study confirms performance status but not age as a major prognostic factor for PFS and OS in recurrent GBM patients initially treated by chemoradiation with TMZ. It also revealed patients with multiple and large lesions to have poorer outcome. Again they confirmed that patients treated with re-resection at relapse do better. Treatment with steroids also appears to be a risk factor for poorer outcome, although this was not specifically addressed in our study.

The most common treatment related toxicity identified in our study was haematological toxicity which was experienced by $31 \%$ patients. Six patients (18\%) developed grade 3/4 thrombocytopenia and 3\% had neutropenic infection. Non-haematological toxicity was identified in $24 \%$. Only $6 \%$ of patients had early treatment termination due to toxicity. It must be noted that retrospective analysis by its nature tends to underestimate the frequency of toxicity but our experience appears to be in line with that of similar studies.

Retrospective analysis by Kuhnhenn et al., ${ }^{11}$ of 57 patients, reported the most common toxicity experienced was CTCAE grade $3 / 4$ haematological toxicity: leucopenia $26 \%$ and thrombocytopenia $26 \%$. Forty three of 131 cycles required dose reduction due to side effects and 21 cycles were delayed and treatment terminated in 7 patients due to unacceptable toxicity. Unlike our experience, Kuhnenn et al. ${ }^{11}$ described significant toxicity and treatment related morbidity with PBV. The authors concluded no benefit of PBV in patients pretreated with $\mathrm{TMZ}$ when compared with much less demanding salvage protocols associated with less toxicity and a better quality of life. This does not appear to be the experience of other studies which showed inferior results from single agent nitrosurea regimens when compared with PCV or PBV. ${ }^{7}$ In their retrospective review of 32 GBM patients treated with ACNU, Happold et al report significantly higher rates of haematological grade $3 / 4(50 \%)(38 \%$ leucopenia and 
$29 \%$ thrombocytopenia) and non-haematological toxicities which led to unsatisfactorily high rates of treatment interruption and dose reduction.

A phase 3 study by Wick comparing single agent Lomustine with Enzastaurin found PFS 6 in lomustine alone treated recurrent GBM patients was $19 \%$ and OS of 28 weeks. The study showed $25 \%$ patients in Lomustine arm developed grade $3 / 4$ thrombocytopenia and $20 \%$ had grade $3 / 4$ neutropenia. $11.9 \%$ patients treated with lomustine received transfusions. Eleven patients treated with lomustine died within 30 days of discontinuation (10 as a result of PD and one as a result of AE).${ }^{16}$ The efficacy and toxicities of single agent Lomustine are very much comparable to PCV combination. Brada et al compared PCV, TMZ (28day cycle) and dose intensified TMZ(21day cycle) in chemotherapy-naive patients with recurrent high grade glioma. The study failed to show any superiority of TMZ over PCV, but demonstrated that the 21-day schedule was inferior to the 28 -day schedule. ${ }^{17}$

Temozolomide rechallenge was explored by Perry et al., ${ }^{18}$ The authors showed that TMZ rechallenge with a continuous $50 \mathrm{mg} /$ $\mathrm{m} 2$ daily schedule is an encouraging approach, especially for patients with recurrence after completion of TMZ administration concurrent with and adjuvant to RT. Few other studies evaluated TMZ rechallenge in TMZ pre-treated patients. ${ }^{19-21}$ Overall median OS ranged from 5.1-13months. But there were various dosing schedules used and importantly few of them were conducted before TMZ and XRT became standard of practice. After recurrence repeat resection and implantation of BCNU-impregnated polymers also prolong survival in selected patients. ${ }^{22}$ A phase II trial using Fotemustine investigated treatment efficacy in a population treated with TMZ and radiotherapy. MGMT methylation status was also assessed. The study demonstrated a PFS6 of $20.9 \%$, and the authors claimed that their study findings represented a benchmark of activity at the time of recurrence. ${ }^{23}$ The same approach has also been investigated by other groups. ${ }^{24,25}$

More recent research hints towards better outcomes with targeted therapies especially antiangiogenic agents. Several phase 2 trials utilising the bevacizumab/irinotecan combination in recurrent Glioblastoma have been conducted. ${ }^{26-29}$ This combination regimen may improve outcome, but overall bevacizumab-based combinations were not superior to historical data obtained with bevacizumab alone.$^{30}$ So far no standard of care with antiangiogenic treatment has been established. We still need more definite information regarding targeted therapies such as the optimal duration of treatment, best chemotherapy combination, dosage and ideal imaging modalities in recurrent Glioblasoma.

Two studies have investigated the combination of daily TMZ and biweekly bevacizumab for recurrent disease ${ }^{31,32}$ but the combination therapy did not show an improvement in outcome compared to single agent. ${ }^{33}$

Response prediction to therapy in patients with recurrent disease remains challenging because of the biological heterogeneity of glioblastoma ${ }^{32}$ as well as numerous other patient-specific factors. The role of O6-methylguanine-DNA methyltransferase (MGMT) status as a predictive marker following relapse remains unclear. No biomarkers of practical use have yet been established. ${ }^{34}$ Nitrourea based chemotherapy still remains a reasonable option in recurrent Glioblastoma patients until more data about novel therapies or their combination with systemic agents becomes available.

\section{Conclusion}

Our experience with the use of PCV as salvage therapy in patients with relapsed GBM following treatment with the Stupp regimen provides evidence of modest survival benefit seemingly consolidating the current consensus from the available literature. However, in contrast to other studies, PCV chemotherapy appears to have been relatively well tolerated with a low risk of serious toxicity.

\section{Acknowledgments}

None.

\section{Conflicts of interest}

Authors declare no conflict of interest. Special Thanks to Rajlakshmi Banerjee for providing editorial assistance of the manuscript.

\section{References}

1. Balana C, Villa S, Teixidor P. Evolution of care for patients with relapsed glioblastoma. Expert rev Anticancer Ther. 2011;11(11):1719-29.

2. Bleeker FE, Molenaar RJ, Leenstra S. Recent advances in the molecular understanding of glioblastoma. J Neurooncol. 2012;108(1):11-27.

3. Stupp R, Mason WP, van den Bent MJ, et al. Radiotherapy plus concomitant and adjuvant temozolomide for glioblastoma. $N$ Engl $J$ Med. 2012;352(10):987-996.

4. Raffaele A, Michele Caraglia M, Serena De Santi, et al. A new schedule of fotemustine in temozolomide-pretreated patients with relapsing glioblastoma. J Neurooncol. 2011;102(3):417-424.

5. Kelly NM, Rimas L, Samir B. Chemotherapy for malignant glioma Expert Review of Neurotherapeutics. 2005;5(Suppl 1):41-49.

6. Wolff JE, Berrak S, Koontz Webb SE, et al. Nitrosurea efficacy in highgrade glioma: a survival gain analysis summarizing 504 cohorts with 24193 patients. J Neurooncol. 2008;88:57-63.

7. Happold C, Roth P, Wick W, et al. ACNU-based chemotherapy for recurrent glioma in the temozolomide era. $J$ Neurooncol. 2009;92(1):45-48.

8. Silvani A, Lamperti E, Gaviani P, et al. Salvage chemotherapy with procarbazine and fotemustine combination in the treatment of temozolomide treated recurrent glioblastoma patients. $J$ Neurooncol. 2008;87(2):143-151.

9. Macdonald DR, Cascino TL, Schold SC, et al. Response criteria for Phase II studies of supratentorial malignant glioma. J Clin Oncol. 1990;8(7):1277-1280

10. Kuhnhenn J, Kowalski T, Steenken S, et al.Procarbazine, carmustine, and vincristine $(\mathrm{PBV})$ for chemotherapy pre-treated patients with recurrent glioblastoma: a single-institution analysis. J Neurooncol. 2012;109(2):433-438.

11. Lamborn KR, Chang M, Prados MD. Prognostic factors for survival of patients with glioblastoma: Recursive partitioning analysis. Neurooncol. 2004;6(3):227-235.

12. Brandes AA, Tosoni A, Amistà $\mathrm{P}$, et al. How effective is BCNU in recurrent glioblastoma in the modern era? A phase II trial. Neurology. 2004;63(7):1281-1284.

13. Schmidt F, Fischer J, Herrlinger U, et al. PCV chemotherapy for recurrent glioblastoma. Neurology. 2006;66(4):587-589.

14. Paccapelo A, Lolli I, Fabrini M G, et al. A retospective pooled analysis of response patterns and risk factors in recurrent malignant glioma patients receiving a nitrosourea based chemotherapy. J Transl Med. 2012;10:90. 
15. Gorlia T, Stupp R, Brandes AA, et al. New prognostic factor and calculators for outcome prediction in patients with recurrent glioblastoma: A pooled analysis of EORTC Brain Tumour Group phase I and II clinical trials. Eur J Cancer. 2012;48(8):1176-1184.

16. Wick W, Puduvalli VK, Chamberlain MC, et al. Phase III study of enzastaurin compared with lomustine in the treatment of recurrent intracranial glioblastoma. J Clin Oncol .28(7):1168-1174.

17. Brada M, Stenning S, Gabe R. Temozolomide Versus Procarbazine, Lomustine, and Vincristine in Recurrent High-Grade Glioma. J Clin Oncol. 2010;28(30):4601-4608.

18. Perry J, Mason W, Belanger K. The Temozolomide RESCUE study: a Phase II trial of continuous (28/28) dose-intense temozolomide (TMZ) after progression on conventional $5 / 28$ day TMZ in patients with recurrent malignant glioma. Clin Oncol. 2010;26(Suppl 15).

19. Kong DS, Lee JI, Kim JH, et al. Phase II trial of low-dose continuous (metronomic) treatment of temozolomide for recurrent glioblastoma. Neuro Oncol. 2010;12(3):289-296.

20. Franceschi E, Omuro AM, Lassman AB, et al. Salvage temozolomide for prior temozolomide responders. Cancer. 2005;104(11):2473-2476.

21. Norden AD, Lesser GJ, Drappatz J, et al. Phase II study of doseintense temozolomide in recurrent glioblastoma. $J$ Clin Oncol. 2013;15(7):930-935.

22. Brem H, Piantadosi S, Burger PC, et al. Placebo-controlled trial of safety and efficacy of intraoperative controlled delivery by biodegradable polymers of chemotherapy for recurrent gliomas. The Polymer-brain Tumor Treatment Group. Lancet. 1995;345(8956):1008-1012.

23. Brandes AA, Tosoni A, Franceschi E, et al. Fotemustine as second-line treatment for recurrent or progressive glioblastoma after concomitant and/or adjuvant temozolomide: a Phase II trial of Gruppo Italiano Cooperativo di Neuro-Oncologia (GICNO). Cancer Chemother. Cancer Chemother Pharmacol. 2009;64(4):769-775.

24. Fabrini MG1, Silvano G, Lolli I, et al. A multi-institutional Phase II study on second-line Fotemustine chemotherapy in recurrent glioblastoma. $J$ Neurooncol. 2008;92(1):79-86.
25. Scoccianti S, Detti B, Sardaro A et al. Second-line chemotherapy with fotemustine in temozolomide-pretreated patients with relapsing glioblastoma: a single institution experience. Anticancer Drugs. 2008;19(6):613-620.

26. Cloughesy TF, Prados MD, Wen PY. A phase II, randomized, noncomparative clinical trial of the effect of bevacizumab (BV) alone or in combination with irinotecan (CPT) on 6-month progression free survival (PFS6) in recurrent, treatment-refractory glioblastoma (GBM). $J$ Clin Oncol. 2008;26(Suppl):91s.

27. Vredenburgh JJ, Desjardins A, Herndon JE, et al. Phase II trial of bevacizumab and irinotecan in recurrent malignant glioma. Clin. Cancer Res. 2007;13:1253-1259.

28. Bokstein F, Shpigel S, Blumenthal DT. Treatment with bevacizumab and irinotecan for recurrent high-grade glial tumors. Cancer. 2008;112(10):2267-2273.

29. Kreisl TN, Kim L, Moore K, et al. Phase II trial of single-agent bevacizumab followed by bevacizumab plus irinotecan at tumor progression in recurrent glioblastoma. JClin Oncol .2009;27(5):740-745.

30. Weller M, Wick W, Hegi ME, et al.Should biomarkers be used to design personalized medicine for the treatment of glioblastoma? Future Oncol. 2010;6(9):1407-1414.

31. Weller M, Cloughesy T, Perry JR, et al.Standards of care for treatment of recurrent glioblastoma--are we there yet? Neuro Oncol. 2013;15(1):4-27.

32. Verhoeff JJ, Lavini C, van Linde ME, et al. Bevacizumab and doseintense temozolomide in recurrent high-grade glioma. Ann Oncol . 2010;21(8):1723-1727.

33. Desjardins A1, Reardon DA, Coan A, et al. Bevacizumab and daily temozolomide for recurrent glioblastoma. Cancer. 2012;118(5):1302-1312.

34. Kesari S. Understanding glioblastoma tumor biology: the potential to improve current diagnosis and treatments. Semin Oncol. 2011;38(suppl 4):S2-S10. 